\title{
Subclinical cerebrovascular disease can impede learning
}

Even in the absence of clinical symptoms, cerebrovascular disease could impair learning ability, according to new research published in Neurology. Cerebrovascular disease is an established risk factor for dementia and it has been previously shown to impair memory. Now, a new cross-sectional study by Hilary Glazer et al. shows that it is also associated with worse verbal learning performance.

Glazer and co-investigators used data from 1,272 communitydwelling participants (mean age 70 years) from The North Manhattan Study, to compare neuroimaging markers of cerebrovascular disease and cerebral volume with verbal learning ability. The selected participants had no prior clinical history of stroke.

In the first trial of a listlearning verbal memory task, the individuals with decreased total cerebral volume (TCV; a marker for brain atrophy due to ageing, neurodegenerative disease or ischaemic damage) and/or increased white matter hyperintensity volume (WMHV; a marker for cerebral small vessel disease) did not differ from those with no evidence for neurodegenerative or cerebrovascular disease.

Over the course of five trials, both low TCV and high WMHV were associated with fewer remembered words. Higher white matter hyperintensity lesion load, but not smaller TCV, was also linked to a flatter learning curve over the verbal learning trials, suggesting that brain atrophy and cerebrovascular disease have distinct effects on learning patterns.

"Our findings suggest that cerebral small vessel disease affects learning processes, with implications for determining phenotypes of vascular cognitive impairment," concludes Clinton
Wright, who led the study. The results demonstrate that verbal learning performance, often included in neuropsychological testing batteries, could be examined in studies of vascular cognitive impairment.

"Many studies have treated memory as if it was a single entity," Wright comments. Performance in an initial learning trial is thought to depend on attention and working memory, whereas subsequent trials engage encoding and consolidation of memory.

\section{4 ...cerebral small vessel disease affects learning processes... 77}

Modelling of verbal list-learning task across trials could help disentangle multiple processes required for learning and memory, which could be attributed to distinct structures and pathways that are differentially susceptible to vascular damage. In future, multimodal imaging studies with fMRI and MEG may also elucidate the effects of vascular lesions on distinct memory processes.

The researchers hope to evaluate the predictive value of imaging markers of cerebrovascular disease and atrophy by examining their association with learning and memory processes in other cohorts. Moreover, a prospective follow-up study could help assess the causal links between subclinical cerebrovascular damage and decline in verbal learning abilities.

Hemi Malkki

Original article Glazer, H. et al. Subclinical cerebrovascular disease inversely associates with learning ability. Neurology doi:10.1212/ WNL.000000000000165

Further reading Rost, N. R. Stroke: More than meets the eye-big consequences of small strokes. Nat. Rev. Neurol. 11, 249-250 (2015) 